\title{
Traduire
}

Revue française de la traduction

$217 \mid 2008$

En territoire terminologique

\section{Le traducteur-terminologue et l'expert, rencontre hypertextuelle}

\section{Michel Rochard}

\section{(2) OpenEdition}

Journals

\section{Édition électronique}

URL : http://journals.openedition.org/traduire/965

DOI : $10.4000 /$ traduire.965

ISSN : 2272-9992

\section{Éditeur}

Société française des traducteurs

\section{Édition imprimée}

Date de publication : 1 juin 2008

Pagination : 61-77

ISSN : 0395-773X

\section{Référence électronique}

Michel Rochard, «Le traducteur-terminologue et l'expert, rencontre hypertextuelle », Traduire [En ligne], 217 | 2008, mis en ligne le 01 juin 2008, consulté le 27 avril 2019. URL : http:// journals.openedition.org/traduire/965; DOI : 10.4000/traduire.965 


\title{
Le traducteur-terminologue et l'expert, rencontre hypertextuelle
}

\author{
MiCHEL ROCHARD \\ Réviseur, $O C D E$
}

Dans cet article, la notion de travail terminologique fera généralement référence au travail de terminologie bilingue ou multilingue. On parlera ensuite d'unité structurée de traduction (UST) pour désigner le service de traduction d'une entreprise commerciale classique, celui d'une administration publique ou encore celui d'une organisation internationale. Le terme traducteur désignera en fait un " traducteur-terminologue " (Rochard, 1992). Par experts, on comprendra les groupes d'auteurs et de lecteurs (ou auditeurs) avertis qui participent à l'élaboration des textes spécialisés(1), à leur examen et à leur critique.

\section{Le travail du traducteur}

Le travail du traducteur dans une UST est, sauf exception, centré sur la transmission du message de la langue source à la langue cible. Le traducteur y est rarement tenu de se soumettre à des impératifs spécifiques liés à un cahier des charges ou à un lectorat. S’il doit y avoir adaptation du message, c'est un travail qui est généralement confié à d'autres intervenants (rédacteurs techniques, services commerciaux, services de communication interne ou externe). Le traducteur dispose donc d'une certaine liberté dans ses choix d'expression, avec en contrepartie la responsabilité de les assumer.

Dans le contexte de l'UST, la traduction pragmatique vise donc à l'équivalence fonctionnelle entre le texte source et le texte cible. L'un

(1) Ces auteurs peuvent certes être des " chercheurs " (scientifiques, juristes, économistes, etc.), mais aussi des analystes dans des sociétés de bourse, des techniciens dans des entreprises industrielles, des cadres commerciaux, etc. 
des principaux moyens mis en œuvre pour ce faire réside dans la maîtrise du discours source («ce qui se conçoit bien, s'énonce clairement ").

La théorie du Sens (Seleskovitch \& Lederer, 1984), la théorie du Skopos (Reiss \& Vermeer, 1991) mais aussi la pragmatique du discours (Moeschler \& Reboul, 1998) vont dans cette direction. La compréhension du sens n'est pas uniquement linguistique. L'équivalence fonctionnelle de la compréhension du discours dans plusieurs langues passe donc entre autres par la compréhension de termes spécifiques à un discours donné, en l'occurrence le discours que doit transmettre le traducteur en langue cible.

Sur ce plan, les théories de la traduction proprement dite donnent sans doute une image trop linéaire du processus de traduction : compréhension-appropriation-réexpression ou plus scolairement lecture, constitution et analyse de corpus documentaire, analyse terminologique, constitution d'un concordancier, etc. jusqu'à la traduction. Ces descriptions ne donnent qu'une image très lisse de la réalité de la traduction. C'est ce qui se passe lorsque tout va bien : quand le discours est connu et maîtrisé par le traducteur, quand la terminologie est établie et coule de source.

En fait, c'est dans les situations de crise qu'il faut observer le travail du traducteur, lorsqu'il se heurte à une difficulté de tout ordre (compréhension du texte source, absence de terminologie établie, difficultés de réexpression, etc.). La rencontre de la difficulté est en fait une constante universelle de l'activité humaine et les moyens de surmonter la difficulté ont été très bien décrits par le philosophe et pédagogue américain John Dewey (Dewey, 1933, 1938). Lorsqu'il se heurte à une difficulté, le traducteur se transforme en enquêteur, à la recherche d'indices, formulant des hypothèses, cherchant à confirmer ses hypothèses par de nouveaux indices et des recoupements, remettant en cause ses propres hypothèses à mesure de la découverte de nouveaux indices jusqu'à parvenir à une solution recevable. Dans ce contexte, le processus 62 de traduction n'a plus rien de linéaire et le traducteur fait au contraire 
flèche de tout bois. Il fait des allers et retours entre documentation, outils terminologiques, mobilisation du bagage cognitif, mise en relation logique des éléments trouvés, etc. Bref, le traducteur s'inscrit dans une démarche hypertextuelle et c'est sans doute ce qui explique pourquoi les traducteurs ont figuré parmi les premiers professionnels ayant totalement intégré l'Internet dans leur travail, y compris lorsqu'ils n'étaient pas des fanatiques de l'informatique.

Si le texte ne pose aucune difficulté, le traducteur n'aura besoin d'aucun outil pour assurer l'équivalence fonctionnelle du texte source et du texte cible. Il n'ouvrira donc aucun dictionnaire, il n'aura recours de manière générale à aucun outil terminologique. En revanche, en situation de difficulté, les outils terminologiques seront là pour apporter des indices ou valider des hypothèses et les différents types d'outils terminologiques favoriseront la résolution de différentes difficultés.

\section{Typologie des outils terminologiques(2)}

Dans la pratique de la traduction et dans son histoire, plusieurs types de terminologie ont été exploités par les traducteurs : le terme à terme, la terminologie contextuelle et la terminologie conceptuelle. Examinons-les un à un afin de pouvoir tirer des leçons pour le programme de travail terminologique du traducteur.

- Le terme à terme : on peut en matière de terme à terme distinguer entre un absolu ( 1 terme source a un équivalent en langue cible et 1 seul) et une pragmatique (à 1 terme et son ou ses synonymes peuvent correspondre 1 ou $n$ équivalents cibles ou traductions). Le terme à terme repose généralement sur une veille terminologique en langue source comme en langue cible, un recensement des équivalents, le tout étant généralement légitimé par l'expérience et par une hiérarchisation des équivalents en fonction de leur caractère attesté ou non. Dans le cadre du terme à terme, le traducteur comme les auteurs de textes en langues source et cible font partie des produc-

(2) Indépendamment des supports techniques. 
teurs de textes(3). L'ensemble des textes constitue le corpus utilisé par le terminologue pour attester les équivalences. Le terme à terme peut avoir plusieurs utilités. Par exemple, lorsque le domaine du texte est bien défini, un glossaire spécialisé portant spécifiquement sur ce domaine va apporter des solutions de traduction qui ont de fortes chances d'être adaptées au contexte et à la phraséologie du domaine. Dans d'autres cas, le traducteur n'aura pas tant besoin d'une terminologie privilégiant les relations de un à un (un terme spécialisé en langue source et un correspondant unique en langue cible), mais de pistes terminologiques (un même terme pourra donner lieu à de multiples solutions de traduction en fonction de la situation dans laquelle il est employé). L'outil terminologique permettra de faire un tri entre ces solutions et donnera des indices qui pourront être validés par une recherche documentaire ou phraséologique sur l'Internet.

- La terminologie contextuelle adopte fondamentalement la même démarche que le terme à terme, sa légitimité supplémentaire auprès des traducteurs vient du fait qu'elle allie terminologie et phraséologie. La somme d'indices apportés au traducteur est donc d'autant plus grande. Les solutions de traduction proposées peuvent être immédiatement testées dans un contexte. Le traducteur y trouve à la fois la solution d'une difficulté terminologique et le mode d'emploi de la solution. Cela étant, il faut bien être conscient que la réalité décrite dans un contexte donné ne coïncidera pas nécessairement et automatiquement avec celle du texte à traduire. Dans ce cas, le terme à terme peut s'avérer plus utile, en permettant au traducteur de s'abstraire du contexte qui lui est proposé et d'ouvrir plus largement le champ des hypothèses terminologiques qu'il va formuler dans son enquête. Un troisième type d'outil peut toutefois apporter des éléments non linguistiques précieux pour la validation de l'enquête terminologique.

(3) Le traducteur se trouve ainsi dans le dilemme de l'œuf et de la poule puisqu'il est à la fois producteur (indirect) de terminologie, utilisateur de terminologie pour la production de nouveaux textes et parfois même critique de la terminologie existante. 
- La terminologie conceptuelle : c'est normalement la "Rolls" des terminologies puisqu'elle tend vers la plus grande rigueur scientifique. Le concept et sa définition assortie d'une source et d'une date sont au centre du travail. Le traducteur va donc y trouver un élément de réflexion logique extrêmement important pour l'enquête au sens où l'entend Dewey. Cela étant, le traducteur devra veiller à la conception de ces outils qui ne doivent pas non plus l'enfermer dans un absolu. Dans l'absolu conceptuel, il existe en effet une définition et une seule d'un concept représenté par un seul et même terme. La définition suffit à légitimer la compréhension et la traduction du terme. On retrouve ici la logique des langues contrôlées. Poussée à l'extrême, cette logique voudrait que si deux définitions d'un même terme sont différentes, c'est que l'une doit être rejetée. Ce serait faire fausse route. Le plus souvent, la difficulté terminologique en traduction correspond précisément à la situation où l'ambiguïté due à la polysémie des mots en langue n'a pas pu être levée en contexte. Il ne faut donc pas se priver des avantages du terme à terme ou de la terminologie contextuelle qui peuvent apporter eux aussi des éléments utiles pour lever l'ambiguiité.

\section{Discours spécialisé et difficultés de traduction}

Le discours spécialisé pose deux difficultés spécifiques de traduction liées à la fois à l'ambiguiité résiduelle des termes spécialisés et aux problèmes de choix terminologiques que cela induit pour le traducteur ainsi qu'à la fonction des termes spécialisés.

Dans le discours spécialisé, les termes spécialisés sont des moyens métonymiques d'assurer l'équivalence fonctionnelle de la compréhension de ce discours pour un groupe d'experts.

Qui dit métonymie dit expression de la réalité sous une forme normalement évocatrice de la réalité, mais aussi sous une forme condensée de cette réalité.

Dans une situation de la vie courante, ces deux caractéristiques n'entravent nullement la communication. Si vous entendez « la porte 
s'il vous plaît " dans le métro, cela vous signale immédiatement qu'une personne demande l'ouverture de la porte parce qu'elle souhaite descendre alors qu'elle ne peut pas actionner elle-même l'ouverture de la porte et qu'il y a des gens qui devront s'écarter pour qu'elle puisse sortir. En revanche, si vous entendez dire en hiver la même expression dans un café où vous venez d'entrer, c'est sans doute que quelqu'un placé près de cette porte et ressentant le froid souhaite que vous fermiez la porte.

Dans le discours spécialisé, la description très condensée de la réalité peut être une source d'ambiguïté terminologique. Prenons l'exemple, du verbe " to leverage » dans le domaine financier. L'image du « levier " que véhicule le terme anglais est assez parlante, car elle renvoie à l'idée qu'emprunter peut être un levier pour "gagner plus ". Mais en même temps, la définition du concept pointe vers la notion d'endettement. Le traducteur devra donc faire un choix entre les notions de «levier" et « d'endettement ». Pour schématiser, lorsque la titrisation avait le vent en poupe, "leverage " renvoyait à cette technique de maximisation de la rentabilité des investissements. En revanche, lorsque les banques cherchent à sortir de la crise des subprimes, elles s'efforcent de réduire leur endettement (leverage). En ce cas, le choix entre ces deux notions va être déterminé par un contexte ayant lui-même fait l'objet d'une analyse logique dans le cadre de l'enquête du traducteur.

On notera par ailleurs que ce rapport entre termes et discours spécialisés est aussi à la base du travail terminologique unilingue et c'est lui qui explique la profusion de glossaires spécialisés sur l'Internet ou dans une organisation internationale comme l'OCDE. Cette profusion est un signe de communication "extravertie ", tournée vers l'extérieur, ce qui facilite normalement la tâche du traducteur.

Mais ce rapport peut être aussi un moyen de connivence exclusive du groupe d'experts à travers le jargon, qui est au contraire un signe de communication " introvertie ", c'est-à-dire confinée au groupe d'experts. 66 Le traducteur va donc devoir apprendre à trier dans la terminologie 
des experts ce qui relève d'une communication « extravertie » et d'une communication " introvertie».

Dans tous ces cas, on voit que la recherche terminologique ne va pas apporter de solution toute faite, mais sera une composante d'un travail d'analyse logique (enquête) qui correspond à la démarche du traducteur face à la difficulté.

\section{Le traducteur entre absolu traductologique et absolu terminologique}

Une autre difficulté du travail terminologique tient pour le traducteur à la nécessité de se garder de deux " absolus ».

- L'absolu traductologique : si l'on pousse à l'extrême la logique de l'équivalence fonctionnelle du discours source spécifique et du discours cible représenté par la traduction de ce discours spécifique (primauté du sens sur la langue), la compréhension du discours source et sa traduction ne devraient pas être influencées par des équivalences terminologiques préétablies. La terminologie serait donc subordonnée à l'équivalence fonctionnelle et il faudrait privilégier la création terminologique en fonction des besoins du texte plutôt que la reprise de terminologies figées dans le texte à traduire qui serait un objet unique.

- L'absolu terminologique : dans cet absolu, la compréhension bilingue du discours nécessite une codification des termes et la relation entre terme source et terme cible doit tendre vers l'univocité, généralement autour d'une définition commune d'un concept, d'une spécification commune d'une pièce ou d'un composant. C'est la logique qui préside aux langues contrôlées (Ryan, 2008).

Le traitement des difficultés de traduction, la navigation entre les écueils des absolus, les avantages et inconvénients respectifs des différents outils terminologiques doivent maintenant être replacés dans le cadre de la démarche du traducteur dont on a vu qu'elle n'était pas linéaire, mais plutôt hypertextuelle. 


\section{Terminologie, concept et traduction hypertextuelle}

Si l'on admet que, face à la difficulté, le traducteur se mue en enquêteur essayant d'avancer vers la résolution de la difficulté au moyen d'indices, d'hypothèses, de recoupements d'indices, de recours à la documentation, à des corpus, aux outils terminologiques et phraséologiques, à ses propres connaissances et à sa propre capacité d'analyse logique, on voit bien que sa démarche est hypertextuelle et non linéaire.

Dès lors, il faut envisager le travail terminologique du traducteur sous cet angle. Nous avons vu que les outils terminologiques fondés sur la définition du concept étaient ceux qui poussaient le plus loin l'analyse de la réalité décrite ou désignée par le terme. Néanmoins, il est en fait très rare que la définition puisse concentrer la totalité des éléments dont a besoin le traducteur pour accomplir sa mission (surmonter les difficultés de la communication), ne serait-ce que parce que la mondialisation induit une évolution permanente du contenu des textes et du champ d'application des termes spécialisés. Les éléments utiles au traducteur peuvent en fait être dispersés dans de multiples composantes de l'outil terminologique : la définition, la source et la date, le pays ou l'institution utilisant le terme, le domaine et le sous-domaine, l'illustration en contexte( ${ }^{(4)}$, les liens vers des documents en ligne, la note technique, les propositions de traduction elles-mêmes et les précisions d'emploi correspondantes, parfois même les éléments grammaticaux. C'est dans cet ensemble d'éléments que se situe le " concept " au sens termino-traductologique. Le traducteur utilise alors ces éléments du concept dans sa démarche hypertextuelle. En effet, chaque situation de difficulté ne nécessite pas forcément la mobilisation simultanée de toutes les composantes du concept. Le traducteur va d'un élément à un autre en fonction de sa recherche d'indices, de ses hypothèses, et de façon plus générale de son enquête. La ventilation des éléments d'information entre différentes zones

(4) Y compris sous forme de schéma pour des termes de technique industrielle ou de mathématiques financières. 
de l'outil terminologique lui apporte alors un gain de temps. Naturellement, les bases de données et outils en ligne facilitent cette ventilation.

\section{Exemple de traitement de l'homonymie par une base de données}

Prenons le cas du terme anglais "consolidation " dans le cadre d'une base de données à spécialisations multiples. Nous pouvons proposer au moins quatre entrées sur ce terme.

- Une première entrée sera juridique : ce terme désignera alors le processus consistant à réunir de nombreuses lois dans le cadre d'un même texte ou code. C'est le processus qui correspond à ce que l'on appelle en France la codification du droit (le code pénal, le code civil, le code des impôts, etc.). Le simple fait que l'outil terminologique indique que cette entrée relève du domaine juridique réduit radicalement le champ des indices de compréhension possibles de ce terme. Le contexte spécifique du texte à traduire rapproché de cette indication thématique suffira à traduire avec une grande certitude ce terme.

- Trois autres entrées sont d'ordre économique : "consolidation " dans le domaine des échanges commerciaux, "consolidation » dans le sens budgétaire et "consolidation " dans le sens managérial et financier. Le premier renvoie à la consolidation des droits de douane qui fait partie des règles du commerce international qui veulent qu'on ne revienne pas sur un engagement de libéralisation des droits de douane dans le cadre de l'OMC. Cette fois, la simple indication du domaine et mieux encore du domaine et de l'institution utilisatrice du terme va permettre de délimiter le concept très rapidement. Dans un contexte budgétaire ("fiscal " en anglais), la notion de "consolidation " va désigner l'assainissement des finances publiques. Enfin, on parlera de "consolidation " pour désigner un mouvement de concentration d'entreprises dans un secteur d'activité, ce qui donnera lieu en comptabilité à des règles de consolidation. 
Est-ce à dire que la définition est inutile et que de simples indications de contexte se suffisent à elles-mêmes ? Certainement pas, car c'est la définition du concept de "consolidation " spécifique à un domaine qui va permettre d'approfondir l'enquête lorsque des zones d'ombre subsistent dans le texte à traduire. On peut en effet se trouver dans un texte aux confins de deux domaines. Ou alors le discours utilisant le terme peut sembler contradictoire avec ce que l'on connaît habituellement de la notion de consolidation.

En effet, comme on l'a vu, c'est lorsque survient la difficulté que l'enquête du traducteur doit aller au-delà des apparences. Or, en dernière analyse, c'est la définition attestée et datée qui va réunir de façon synthétique le maximum d'éléments consignés dans l'outil terminologique pour comprendre le texte ou lever les ambiguïtés et contradictions.

\section{Le traducteur et l'expert}

Selon Dewey, le déclenchement d'une enquête est une réaction naturelle de l'homme confronté à une difficulté, à une situation inconnue. C'est dans ces situations que le traducteur va mobiliser tous les outils dont il dispose, dont l'outil terminologique. Cela étant, dès lors qu'on consigne un terme/concept dans un outil terminologique, c'est qu'il a déjà basculé dans le connu. Or, ce qui fait la difficulté de son métier, c'est que le traducteur ne traduit pas tant pour relater le connu que pour aller au-delà du connu, pour faire comprendre ce qui n'est pas (ou mal) connu, ce qui n'est pas (ou mal) compris. C'est précisément là que se situe le point de rencontre du traducteur et de l'expert. Le traducteur doit s'approprier le texte à traduire et sa part d'inconnu pour transmettre le discours ; l'expert doit, en tant qu'auteur éventuel, comprendre et analyser le réel dans son évolution et sa nouveauté pour le faire comprendre à travers son propre discours ou, en tant que spécialiste, comprendre le discours initial pour se l'approprier, l'analyser, le développer, le critiquer ou encore le contredire. L'expert et le traducteur travaillent donc à la pointe des savoirs, à la lisière mouvante de 
l'inconnu. L'un comme l'autre a besoin à un moment donné d'intellectualiser ou de conceptualiser l'inconnu, de le ramener à des termes linguistiquement et contextuellement maniables, repérables et isolables. Ils se comportent tous les deux comme des terminologues, l'un cherchant à définir le concept pour le décrire et le commenter, l'autre cherchant à s'assurer de sa "définition " à l'aide de divers éléments pour mieux transmettre le discours dans une autre sphère linguistique.

Lorsque l'enquête du traducteur se heurte à des lacunes de l'outil terminologique et de la documentation, l'expert peut être un recours précieux en apportant de nouveaux éclairages, du liant entre terme, définition, domaine, zone d'utilisation, contexte, phraséologie, traduction, etc. Le traducteur ne doit donc pas hésiter à aller vers l'expert, à exposer la difficulté, ce qu'il fera d'autant mieux qu'il aura poussé sa propre enquête au maximum de ses moyens.

Mais la rencontre du traducteur et de l'expert ne s'arrête pas là et, si les conditions le permettent, elle doit être prolongée en l'inscrivant dans le programme de travail terminologique. Les conditions naturellement les plus propices à cette rencontre et à ses prolongements correspondent à la situation d'une entreprise, d'une organisation ou d'une administration dotée d'une UST.

\section{L'unité structurée de traduction et les experts : scénarios de travail terminologique commun}

Pour une UST, la constitution d'un patrimoine terminologique est un moyen d'assurer sa pérennité, de transmettre les savoirs d'une génération de traducteurs à l'autre. À l'heure de la mondialisation, de la transversalité des problèmes (économie, écologie, politique, droit, science, morale, santé, etc.), le programme de travail terminologique ne doit pas se contenter d'un recensement de termes et de traductions. Il doit viser plus haut pour constituer une véritable base de connaissances permettant aux traducteurs en particulier d'optimiser leurs recherches documentaires et terminologiques. La recherche du plus 
grand nombre d'éléments possibles permettant de représenter le concept, y compris les définitions en langue source ou cible et la structuration de ces éléments à travers différents champs terminologiques procèdent de ce souci d'optimisation des démarches.

Mais les gains d'efficience ne s'arrêtent pas là. En effet, au sein de l'entité dotée d'une UST, les experts sont confrontés eux-mêmes à des problèmes de terminologie. S'ils veulent être parties prenantes aux débats entre experts, ils doivent eux-mêmes savoir de quoi ils parlent, en d'autres termes, ils doivent définir les concepts qu'ils utilisent. C'est ce qui explique la multiplication des glossaires en marge ou dans le cadre de travaux de recherche et de publications (y compris dans des rapports annuels d'entreprise). Dans le cadre de ces projets, il n'est pas rare que les experts prévoient de construire un glossaire qui sera annexé à une publication ou qu'ils le construisent dès les prémices du projet pour communiquer entre eux.

Mettre la base de connaissances de l'UST au service des experts : il est fréquent que les experts (dont les effectifs se renouvellent et qui peuvent ne pas être au fait de l'histoire de l'entité) réinventent à cette occasion des définitions qui pouvaient exister par ailleurs dans l'entité. Et c'est là que les traducteurs peuvent apporter leur pierre en amont de la réalisation du projet, notamment si leurs outils terminologiques consignent de nombreuses définitions de termes. Au-delà du conseil ponctuel apporté par l'expert au traducteur, il y a là un premier scénario de rencontre qui peut avoir des répercussions utiles pour l'élément principal du programme de travail de l'UST, à savoir la réalisation et l'entretien de sa base de connaissances.

Contribuer à la formation des experts : l'existence d'une base commune de connaissances au sein de l'entité peut aussi contribuer à la formation de nouveaux experts et à l'intégration d'experts participant ponctuellement à des travaux. Il est en effet important que tous les experts parlent des mêmes notions. C'est d'autant plus important dans le cas d'une entité internationale dans laquelle les influences nationales peuvent brouiller la communication. 
Déjouer ensemble les pièges terminologiques : il arrive aussi, dans un cadre international, que les experts eux-mêmes se laissent piéger par l'apparence linguistique des termes et pensent se trouver face à un concept spécifique alors que seul l'habillage linguistique du concept était nouveau. Cela a été le cas par exemple de la notion de "tertiary education" que l'on a longtemps rendue en français par "enseignement tertiaire " avant que les traducteurs eux-mêmes, par leur travail de veille terminologique, rapprochent les concepts français et anglais pour aboutir à une équivalence plus parlante en français entre "tertiary education " et "enseignement supérieur ». De même, c'est la rencontre des experts et des traducteurs dans une situation de quiproquo extrême qui a permis de rectifier la terminologie française des adjudications de titres d'État (Rochard, 1991).

Intégrer les traducteurs à l'élaboration des grands projets de l'entité : le travail terminologique associant très en amont experts et traducteurs est cette fois indispensable au moment de la rédaction et de la traduction de textes de référence majeurs pour une entité, en particulier une organisation internationale. Il permet de lever les ambiguités, d'éviter que les choix terminologiques intuitifs du traducteur se heurtent à des impasses dans la suite de sa traduction. Il sensibilise les experts à l'impératif de clarté de leur texte. En outre, si le texte doit donner lieu à un glossaire, il sera bien plus facile d'assurer la cohérence générale de ce glossaire qui pourra ensuite être intégré à la base de connaissances et servir de référence à d'autres experts comme à d'autres traducteurs.

Publier ensemble des travaux terminologiques: la nécessité d'assurer le rayonnement des compétences des experts et la visibilité de l'entité peuvent aboutir à la publication de travaux terminologiques. L'OCDE le fait par exemple dans le cadre de programmes d'ouverture vers les nouveaux acteurs de l'économie mondiale, ces fameux " pays émergents ", ou vers les pays en développement qui ont besoin d'une assistance technique et d'outils de formation pour leurs propres experts. La présence d'une base de connaissances bilingue organisée sur le plan thématique notamment revêt alors toute son utilité. Elle apporte un 
formidable gain de temps pour les experts comme pour les traducteurs et permet de mettre en évidence leurs compétences respectives.

Mettre la base de connaissances à la disposition du public : si le programme de travail terminologique des traducteurs devient, grâce à la collaboration avec les experts, une composante du programme de travail de l'entité, il n'est plus exclu de diffuser la base de connaissances dans le grand public, selon les modalités économiques adaptées à chaque entité.

\section{Quelques réflexions rapides sur la faisabilité du programme de travail terminologique, en guise de conclusion}

À travers ces scénarios, nous venons de présenter une ébauche du programme de travail terminologique d'une UST en lien étroit avec les experts de l'entité correspondante. Il convient de souligner qu'un tel programme est d'autant plus facilement réalisable que nous disposons aujourd'hui de langages informatiques, de logiciels et de matériels tout à fait adaptés à ses besoins. Il est donc possible de faire coïncider l'architecture de la base de connaissances avec la démarche hypertextuelle des traducteurs, ce qui ne peut que les aider à faire leur travail de façon efficiente.

Le lecteur peut néanmoins légitimement s'interroger sur la faisabilité d'un tel programme de travail. Il aura d'autant plus raison que les ressources humaines et financières de bien des UST tendent malheureusement à diminuer. Cela étant, nombre d'exemples tendent à prouver le contraire :

- La prolifération d'énormes glossaires sur l'Internet (dans les domaines de la finance ou des assurances par exemple);

- Le succès de Termium (Gouvernement canadien) ou du Grand dictionnaire terminologique (Québec) ;

- La référence que constitue le Dictionnaire de la comptabilité et de la gestion financière de Louis Ménard, réalisé en collaboration avec des experts-comptables belges, canadiens et français ; 
- La base de données terminologiques de la Banque de France à la fin des années 80 et les deux tomes du Dictionnaire économique de l'anglais et du français qu'elle a permis de réaliser en collaboration entre les experts et les traducteurs de cette institution ;

- Le troisième tome de ce même projet réalisé et publié avec le ministère français de l'Économie et des Finances qui a mobilisé des experts de ces deux institutions, mais aussi de la Cour des comptes, des ambassades de France en Grande-Bretagne et aux États-Unis, etc. et qui est certainement l'un des meilleurs ouvrages comparatifs sur les finances publiques des trois pays.

On pourrait en citer bien d'autres qui tendent à légitimer un tel programme. Certes, dans des entités dotées d'UST, la réalisation de tels projets suppose une démarche volontariste, mais ces projets répondent aussi à des besoins réels et convergents des experts, des traducteurs et des entités elles-mêmes. Dans le contexte actuel difficile que connaissent les UST, ces projets apparaissent comme un vecteur de plus en plus indispensable de développement des compétences des traducteurs et d'amélioration de leur image auprès des experts et du grand public. Enfin, ce sont d'excellentes occasions d'amener les traducteurs à sortir de leur "bulle " pour mieux affronter les évolutions futures de leur métier.

Michel Rochard est titulaire d'un diplôme de traduction de l'Université de Mayence à Germersheim et d'un doctorat de traductologie de l'Université de Paris 3 (ESIT), réviseur à la Division de la traduction de l'Organisation de coopération et de développement économiques $(O C D E)$ et chargé de cours de traduction économique et financière à l'Université de Paris 7 (EILA). 


\section{Bibliographie :}

Banque de France, Dictionnaire économique de l'anglais et du français, Collection Documentation et information, vol. I et II, Paris, Economica, 1991-1992.

Dewey J., How we think, Boston, D.C. Heath \& Co, 1933.

Dewey J., "Logic: The theory of inquiry", in : J.A. Boydston (éd.), John Dewey: The later works, 1925-1953., Vol 12, 1938, Carbondale, IL, Southern Illinois University Press, 1986.

Ménard L., Dictionnaire de la comptabilité et de la gestion financière, 2e édition, Institut canadien des comptables agréés, 2004.

Ministère de l'Économie et des Finances \& Banque de France, Dictionnaire économique de l'anglais et du français, Budget et financement de l'État (France, Royaume-Uni et États-Unis), volume hors série, La Documentation française, 1997.

Moeschler J. et Reboul A., Pragmatique du discours : de l'interprétation de l'énoncé à l'interprétation du discours, Paris, Armand Colin, 1998.

Reiß, K. et Vermeer H., Grundlegung einer allgemeinen Translationstheorie, 2. Auflage, Tübingen, Francke, 1991.

Rochard M., "À propos de la terminologie des méthodes d'adjudication ", Association française des Banques, Revue Banque $\mathrm{n}^{\circ} 518,1991$.

Rochard M., "Le traducteur face à la terminologie : consommateur ou acteur ?", Commission des Communautés européennes, Revue Terminologies et Traduction, $\mathrm{n}^{\circ}$ 2/3, 1992.

Rochard M., " La rétroconception entre compréhension et ré-expression des textes économiques ", in : Élisabeth Lavault-Olléon (éd.), Traduction spécialisée : pratiques, théories, formations, travaux interdisciplinaires et plurilingues en langues étrangères appliquées, vol. 10, Peter Lang, Berne, 2006. 
Ryan R., "Les langues contrôlées sont-elles l'avenir de la traduction ? ", in : Froeliger Nicolas (éd.), La traductologie de plein champ (Vol. II), La Tribune Internationale des langues vivantes (à paraître), 2008.

Seleskovitch D., Langages, langues et mémoire, Paris, Minard, coll. Lettres Modernes, 1975.

Seleskovitch D., Traduire : les idées et les mots, Études de Linguistique Appliquée $\mathrm{n}^{\circ}$ 24, Paris, Didier, 1976.

Seleskovitch D., Lederer M., Interpréter pour traduire, Paris, Didier Érudition, 1984. 